\title{
Health public issues and socioeconomic status.
}

\author{
Suhasini Salivendra* \\ *Department of Biotechnology, Bhopal University, Madhya Pradesh, India
}

Accepted on September 18, 2020

\section{Commentary}

According to the World Health Organization (WHO), Health is define as people have complete physical, mental and social well-being and absence of disease linked to the way people live their lives. Some experts suggest that health is the physical fitness and mental stability but, health definition is different from person to person.

Some Research work shows that there is a correlation between health and wealth, where people in the upper class persons have better health and they can avoid ill-health and living longer than those in the lower classes persons. As a result, mortality rates are greater for the lower social classes than for the higher social classes. Researchers are that demonstrates that mortality rate and health status is totally depends on individual income, education, occupational and racial differences. In the world policy is applicable for low income people who are living below poverty. The contributing factors for mortality and health issues such as poor diet, obesity, drug and alcohol consumption. Factors like language, age, financial barriers and geographical location can affect the poverty. A geographical barrier can be that some patients may have to travel long distances for certain services or to receive specific treatments.

According to $\mathrm{WHO}$, those people who are living in slum areas and even they do not have minimum income for living, categorized into poverty. People, who are living under poverty, are with adequate housing, nutrition, and clothing. People who live in poverty are less to go to clinics and hospitals or health screening opportunities.

The government should implement new schemes for below poverty people for their health benefits, education and for household purpose. They should appoint health care workers and nurse with necessary skills and adequate knowledge in order to educate children and parents who are living under poverty. The necessary resources like 24 hours ambulance availability and trained nurses should be available in that communication and education process. Some evidence suggests that when they approach children and families about health and education, that problem-solving techniques some success like preventing diseases and premature deaths.

Some diseases commonly occur according to the race and socioeconomic group. For example Hypertension mostly seen in blacks than white persons. In some countries, exposure to sunlight is very low, in such cases skin cancer rates are high than other countries. The number is depending on the type of diseases and the number of population exposure to the sun light. Some diseases are epidemic and some diseases endemic. Endemic diseases are constantly present in community or population like Acquired Immunodeficiency syndrome.

Epidemiology is a technique is using to analyze the incidence, distribution, determinants and control of diseases or health status. Many terms are used to define public health issues, risk factors and causes of diseases. Out of these Morbidity, Mortality and Incidence. Morbidity is defined as the total number of people suffering from disease at a specific time of interval. Mortality is defines as the total number of people is died from specific disease.

Some diseases commonly occur according to the race and socioeconomic group. For example Hypertension mostly seen in blacks than white persons. In some countries, exposure to sunlight is very low, in such cases skin cancer rates are high than other countries. The number is depending on the type of diseases and the number of population exposure to the sun light. Some diseases are epidemic and some diseases endemic. Endemic diseases are constantly present in community or population like Acquired Immunodeficiency syndrome.

Epidemiology is a technique is using to analyze the incidence, distribution, determinants and control of diseases or health status. Many terms are used to define public health issues, risk factors and causes of diseases. Out of these Morbidity, Mortality and Incidence. Morbidity is defined as the total number of people suffering from disease at a specific time of interval. Mortality is defines as the total number of people is died from specific disease. The other two commonly used to measure the diseases are Prevalence and Incidence.

\section{*Correspondence to:}

\section{Suhasini Salivendra}

Department of Biotechnology

Bhopal University

Madhya Pradesh

India

E-Mail: salivendra_suhasini@rediffmail.com 